\title{
SPECTRAL MULTIPLIERS OF LAPLACE TRANSFORM TYPE FOR THE LAGUERRE OPERATOR
}

\author{
Emanuela Sasso
}

\begin{abstract}
We shall work with the Laguerre measure on $\mathbf{R}_{+}^{d}$ and the associated Laplacian $\mathcal{L}_{\alpha}$, by means of which the Laguerre semigroup is defined. Our main result is a multiplier theorem, saying that a function of $\mathcal{L}_{\alpha}$ which is of Laplace transform type defines an operator of weak type $(1,1)$ for the Laguerre measure. Our starting point is the well-known relationship between the Laguerre and Ornstein-Uhlenbeck semigroups.
\end{abstract}

\section{INTRODUCTION}

The purpose of this paper is to study the weak type $(1,1)$ boundedness of a special class of spectral multipliers for the Laguerre operator $\mathcal{L}_{\alpha}$, whenever $\alpha=\left(\alpha_{1}, \ldots, \alpha_{d}\right)$ is a multi-index with $\alpha_{i} \geqslant 0$, for each $i=1, \ldots, d$.

The operator $\mathcal{L}_{\alpha}$ is a self-adjoint "Laplacian" on $L^{2}\left(\mu_{\alpha}\right)$, where $\mu_{\alpha}$ is the Laguerre measure of type $\alpha$ on $\mathbf{R}_{+}^{d}$, that is, $\mathrm{d} \mu_{\alpha}(x)=\prod_{i=1}^{d}\left(x_{i}^{\alpha_{i}} e^{-x_{i}} / \Gamma\left(\alpha_{i}+1\right)\right) \mathrm{d} x$ on $\mathbf{R}_{+}^{d}=\{x$ $\in \mathbf{R}^{d}: x_{i}>0$ for each $\left.i=1, \ldots, d\right\}$. It is well known that the spectral resolution of $\mathcal{L}_{\alpha}$ is

$$
\mathcal{L}_{\alpha}=\sum_{n=0}^{\infty} n \mathcal{P}_{n}^{\alpha}
$$

where $\mathcal{P}_{n}^{\alpha}$ is the orthogonal projection on the space spanned by Laguerre polynomials of total degree $n$ and type $\alpha$ in $d$ variables (see, for instance, [12]). The operator $\mathcal{L}_{\alpha}$ is the infinitesimal generator of a "heat" semigroup, called the Laguerre semigroup, $\left\{e^{-t \mathcal{L}_{\alpha}}\right.$ : $t \geqslant 0\}$, defined in the spectral sense as

$$
e^{-t \mathcal{L}_{\alpha}}=\sum_{n=0}^{\infty} e^{-n t} \mathcal{P}_{n}^{\alpha}
$$

It can be shown that for each $t>0, e^{-t \mathcal{L}_{\alpha}}$ is an integral operator, whose kernel with respect to the Laguerre measure is

$$
m_{\alpha, t}(x, y)=\left(1-e^{-t}\right)^{-|\alpha|-d} \int_{[-1,1]^{d}} e^{(1 / 2)\left(q_{+}(x, y, s) / e^{t / 2}+1\right)-(1 / 2)\left(g_{-}(x, y, s) / e^{t / 2}-1\right)} \Pi_{\alpha}(s) \mathrm{d} s
$$

Received 4th August, 2003

Copyright Clearance Centre, Inc. Serial-fee code: 0004-9727/04 \$A2.00+0.00. 
where

$$
\begin{aligned}
q_{ \pm}(x, y, s) & =\sum_{i=1}^{d}\left(x_{i}+y_{i} \pm 2\left(x_{i} y_{i}\right)^{1 / 2} s_{i}\right), \\
\Pi_{\alpha}(s) & =\prod_{i=1}^{d} \frac{\Gamma\left(\alpha_{i}+1\right)}{\Gamma\left(\alpha_{i}+1 / 2\right) \sqrt{\pi}}\left(1-s_{i}^{2}\right)^{\alpha_{i}-1 / 2},
\end{aligned}
$$

and $|\alpha|=\alpha_{1}+\cdots+\alpha_{d}$. See, for instance, [1] and [12].

A line of research is devoted to the study of the boundedness properties of several operators naturally associated with self-adjoint operators. As a significant but incomplete sample of these investigations about the Laguerre operator, we can cite the papers [1, $3,8]$ and our recent article [9]. In particular, the present work completes the study of the functional calculus associated to the Laguerre operator of [9], by considering the multipliers of Laplace transform type (in the terminology of [11]).

The assumption that a function $M$ is of Laplace transform type implies that $M$ extends to a holomorphic function on the right half plane $\{z: \Re z>0\}$, which is bounded on every sector $\mathbf{S}_{\theta}=\{z \in \mathbf{C}:|\arg z|<\theta\}$, with $0<\theta<\pi / 2$. It follows from the general Littlewood-Paley theory for semigroups that, if $M$ is of Laplace transform type, then $M\left(\mathcal{L}_{\alpha}\right)$ is bounded on all spaces $L^{p}\left(\mu_{\alpha}\right)$, with $1<p<\infty$ (see [11]).

In [6], the author gave a sufficient condition for the existence of a non-holomorphic functional calculus for the generator $A$ of a symmetric contraction semigroup on $L^{p}$, $1 \leqslant p \leqslant \infty$. He proved that if the norms of the imaginary powers $A^{i u}$ of a generator, as an operator on $L^{p}$, grow at most exponentially as $|u|$ tends to infinity, then the multiplier operator $M(A)$ is bounded on $L^{p}$, provided that $M$ extends to a bounded and holomorphic function on a fixed sector $\mathbf{S}_{\boldsymbol{\theta}}$, satisfying a Hörmander condition of a sufficiently high order on the boundary of the sector. A standard method to obtain estimates of the norms of $A^{i u}$ on $L^{p}$ is via complex interpolation between a weak type $(1,1)$ estimate and the $L^{2}$ estimate. Observe that the $L^{2}$ estimate is trivial, since by the spectral theorem the operators $A^{i u}$ are unitary on $L^{2}$.

In view of these remarks it is important to obtain sharp estimates of the weak type $(1,1)$ quasinorm of the imaginary powers of the operator $\mathcal{L}_{\alpha}$ (see also [9]). This is a particular case of a multiplier operator $M\left(\mathcal{L}_{\alpha}\right)$ of Laplace transform type, to which we may apply our main result.

The paper is organised as follows. Section 2 contains the setup and the derivation of the kernel of the multiplier operator outside the diagonal, in terms of an integral involving the kernel of the Laguerre semigroup. Finally, in Section 3, we prove the weak type $(1,1)$ estimate for the spectral multipliers of Laplace transform type. 


\section{Multipliers of LAPLACE TRANSFORM TYPE}

In this section, we introduce the particular class of spectral multipliers of interest, that is, the multipliers of Laplace transform type. We say that a function $M$ is of Laplace transform type if

$$
M(k)=k \int_{0}^{\infty} \phi(t) e^{-k t} \mathrm{~d} t, \quad k>0,
$$

where $\phi$ is a bounded measurable function on $\mathbf{R}_{+}$. Changing variables $e^{-t}=r$, we observe that a function $M$ is of Laplace transform type if and only if

$$
M(k)=k \int_{0}^{1} \psi(r) r^{k} \frac{\mathrm{d} r}{r}, \quad k>0,
$$

where $\psi(r)=\phi(-\log r)$. By spectral theory, the multiplier operator

$$
M\left(\mathcal{L}_{\alpha}\right)=\sum_{n=1}^{\infty} M(n) \mathcal{P}_{n}^{\alpha}
$$

is a bounded operator on $L^{2}\left(\mu_{\alpha}\right)$. Moreover, it follows from the general Littlewood-Paley theory for semigroups that, if $M$ is of Laplace transform type, then $M\left(\mathcal{L}_{\alpha}\right)$ is bounded on all spaces $L^{p}\left(\mu_{\alpha}\right), 1<p<\infty$ (see [11]). In the next section, we shall show that $M\left(\mathcal{L}_{\alpha}\right)$ is also of weak type $(1,1)$ with respect to the Laguerre measure.

The advantage of working with multipliers of this type is that, by spectral theory, $M\left(\mathcal{L}_{\alpha}\right)$ may be written by means of the Laguerre semigroup. Therefore we get, at least formally, an explicit expression of the action of $M\left(\mathcal{L}_{\alpha}\right)$ in terms of the heat kernel $m_{\alpha, t}$. To be more specific, by the spectral theorem and by $(2.1)$, we have

$$
M\left(\mathcal{L}_{\alpha}\right)=\int_{0}^{1} \psi(r) \mathcal{L}_{\alpha} r^{\mathcal{L}_{\alpha}} \frac{\mathrm{d} r}{r},
$$

where the integral converges in the weak operator topology of $L^{2}\left(\mu_{\alpha}\right)$. Indeed, if for every pair of functions $f, g$ in $L^{2}\left(\mu_{\alpha}\right)$, we denote their inner product in $L^{2}\left(\mu_{\alpha}\right)$ by $\langle f, g\rangle_{\mu_{\alpha}}$, we have that

$$
\begin{aligned}
\left\langle M\left(\mathcal{L}_{\alpha}\right) f, g\right\rangle_{\mu_{\alpha}} & =\sum_{k=1}^{\infty} M(k)\left\langle\mathcal{P}_{k}^{\alpha} f, g\right\rangle_{\mu_{\alpha}} \\
& =\sum_{k=1}^{\infty} k \int_{0}^{1} \psi(r) r^{k} \mathrm{~d} r / r\left\langle\mathcal{P}_{k}^{\alpha} f, g\right\rangle_{\mu_{\alpha}}
\end{aligned}
$$

Since $\sum_{k=0}^{\infty}\left|\left\langle\mathcal{P}_{k}^{\alpha} f, g\right\rangle_{\mu_{\alpha}}\right| \leqslant\|f\|_{2}\|g\|_{2}$, we may interchange the order of summation and integration to get

$$
\begin{aligned}
\left\langle M\left(\mathcal{L}_{\alpha}\right) f, g\right\rangle_{\mu_{\alpha}} & =\int_{0}^{1} \psi(r) \sum_{k=1}^{\infty} k r^{k}\left\langle\mathcal{P}_{k}^{\alpha} f, g\right\rangle_{\mu_{\alpha}} \mathrm{d} r / r \\
& =\int_{0}^{1} \psi(r)\left\langle\mathcal{L}_{\alpha} r^{\mathcal{L}_{\alpha}} f, g\right\rangle_{\mu_{\alpha}} \mathrm{d} r / r
\end{aligned}
$$


as required.

If $M$ is of Laplace transform type, then $M\left(\mathcal{L}_{\alpha}\right)$ is a continuous operator from the space of test functions to the space of distributions on $\mathbf{R}_{+}^{d}$ and so it has a distributional kernel. In the following, we shall deduce by (2.2) that, off the diagonal, this kernel agrees with a function $K_{\psi}$, satisfying gradient estimates in a suitable neighbourhood of the diagonal.

The first tool in the proof of our main result is the relationship between the Laguerre and Ornstein-Uhlenbeck semigroups. It is well known that for special values of $\alpha$ the analysis of the Laguerre semigroup can be interpreted as the analysis of the OrnsteinUhlenbeck semigroup acting on radial functions (see [1] and [3]). As in [5], the idea is to decompose $M\left(\mathcal{L}_{\alpha}\right)$ into two operators, one given by a kernel supported off the diagonal, and the other satisfying "standard" gradient estimates in a suitable neighbourhood of the diagonal.

First, it is convenient to perform a change of coordinates in $\mathbf{R}_{+}^{d}$. If $x=\left(x_{1}, \ldots, x_{d}\right)$ is a vector in $\mathbf{R}_{+}^{d}$, then $x^{2}$ denotes the vector $x^{2}=\left(x_{1}^{2}, \ldots, x_{d}^{2}\right)$. Let $\Psi: \mathbf{R}_{+}^{d} \rightarrow \mathbf{R}_{+}^{d}$ be defined by $\Psi(x)=x^{2}$ and let $\widetilde{\mu}_{\alpha}=\Psi^{*} \mu_{\alpha}$ be the pull-back of the measure $\mu_{\alpha}$. Then $\widetilde{\mu}_{\alpha}$ is the the probability measure

$$
\mathrm{d} \widetilde{\mu}_{\alpha}(x)=2^{d} \prod_{i=1}^{d} \frac{x_{i}^{2 \alpha_{i}+1} e^{-x_{i}^{2}}}{\Gamma\left(\alpha_{i}+1\right)} \mathrm{d} x,
$$

on $\mathbf{R}_{+}^{d}$. The map $f \rightarrow \mathcal{U}_{\Psi} f=f \circ \Psi$ is an isometry of $L^{q}\left(\mu_{\alpha}\right)$ onto $L^{q}\left(\widetilde{\mu}_{\alpha}\right)$, for every $q$ in $[1, \infty]$. Let $\widetilde{\mathcal{L}}_{\alpha}$ denote the operator $\mathcal{U}_{\Psi} \mathcal{L}_{\alpha} \mathcal{U}_{\Psi}^{-1}$. We may reduce the problem to the study of the weak type $(1,1)$ boundedness of the operator $M\left(\widetilde{\mathcal{L}}_{\alpha}\right)$, with respect to the measure $\tilde{\mu}_{\alpha}$.

It is easy to observe that $r^{\tilde{\mathcal{L}}}$, with $0 \leqslant r<1$, may be written as an integral operator, whose kernel is

$$
\widetilde{m}_{\alpha, r}(x, y)=\int_{[-1,1]^{d}} \widetilde{m}_{\alpha, r}(x, y, s) \mathrm{d} s,
$$

where

$$
\tilde{m}_{\alpha, r}(x, y, s)=(1-r)^{-|\alpha|-d} \exp \left(-\frac{q_{-}\left(r x^{2}, y^{2}, s\right)}{1-r}\right) \Pi_{\alpha}(s),
$$

with respect to the measure $m_{\alpha}$, that is, the probability measure on $\mathbf{R}_{+}^{d}$ defined by

$$
\mathrm{d} m_{\alpha}(x)=2^{d} \prod_{i=1}^{d} \frac{x_{i}^{2 \alpha_{i}+1}}{\Gamma\left(\alpha_{i}+1\right)} \mathrm{d} x .
$$

The expression for $\tilde{m}_{\alpha, r}$ is obtained from (1.1) by using the change of variable $t$ $=-\log r$. Since the kernel $\tilde{m}_{\alpha, e^{-t}}$ satisfies the heat equation $\partial_{t} u=-\tilde{\mathcal{L}}_{\alpha} u$, it follows that $r \partial_{r} \tilde{m}_{\alpha, r}=\tilde{\mathcal{L}}_{\alpha} \tilde{m}_{\alpha, r}$. 
For each $\psi \in L^{\infty}\left(\mathbf{R}_{+}\right)$, define

$$
K_{\psi}(x, y)=\int_{[-1,1]^{d}} K_{\psi}(x, y, s) \mathrm{d} s
$$

with

$$
K_{\psi}(x, y, s)=\int_{0}^{1} \psi(r) \partial_{r} \widetilde{m}_{\alpha, r}(x, y, s) \mathrm{d} r .
$$

In order to define the "local" region, we consider the extra variable $s$ in $[-1,1]^{d}$. This artifice is suggested by the description of the local region in polar coordinates in the Ornstein-Uhlenbeck case, and by the form of the kernel $\tilde{m}_{\alpha, r}$. For each $\tau>0$ the local region $N_{\tau}$ is the set

$$
\left\{(x, y, s) \in \mathbf{R}_{+}^{d} \times \mathbf{R}_{+}^{d} \times[-1,1]^{d}: q_{-}\left(x^{2}, y^{2}, s\right)^{1 / 2} \leqslant \frac{\tau}{1+|x|+|y|}\right\} .
$$

LEMMA 1. If $x \neq y$ the integral $K_{\psi}(x, y)$ converges absolutely. Moreover, in this case there exists a constant $C$ such that

$$
\begin{gathered}
\left|K_{\psi}(x, y, s)\right| \leqslant C\|\psi\|_{\infty} q_{-}\left(x^{2}, y^{2}, s\right)^{-|\alpha|-d} \Pi_{\alpha}(s), \\
\left|\nabla_{(x, y)} K_{\psi}(x, y, s)\right| \leqslant C\|\psi\|_{\infty} q_{-}\left(x^{2}, y^{2}, s\right)^{-|\alpha|-d-1 / 2} \Pi_{\alpha}(s),
\end{gathered}
$$

whenever $(x, y, s) \in N_{\tau}$.

PROOF: By a straightforward calculation we have that

$$
\partial_{r} \widetilde{m}_{\alpha, r}(x, y, s)=(1-r)^{-|\alpha|-d-2} e^{-\left(q_{-}\left(r x^{2}, y^{2}, s\right) / 1-r\right)} P(\sqrt{r}) r^{-1 / 2} \Pi_{\alpha}(s),
$$

where $P(\sqrt{r})$ is a polynomial in $\sqrt{r}$ of degree three, whose coefficients depend on $x, y, s$. Thus the function $r \mapsto \partial_{r} \widetilde{m}_{\alpha, r}(x, y, s)$ changes sign at most a finite number of times. It follows that there exists a constant $C$ such that

$$
\begin{aligned}
\int_{0}^{1}\left|\psi(r) \partial_{r} \widetilde{m}_{\alpha, r}(x, y)\right| \mathrm{d} r & \leqslant \int_{0}^{1} \int_{[-1,1]^{d}}\left|\psi(r) \partial_{\tau} \tilde{m}_{\alpha, r}(x, y, s)\right| \mathrm{d} r \mathrm{~d} s \\
& \leqslant C\|\psi\|_{\infty} \int_{[-1,1]^{d}} \max _{0 \leqslant r<1} \tilde{m}_{\alpha, r}(x, y, s) \mathrm{d} s<\infty
\end{aligned}
$$

for all $x$ and $y$ in $\mathbf{R}_{+}^{d}$. We claim that in the local region $N_{\tau}$ the following holds

$$
q_{-}\left(r x^{2}, y^{2}, s\right) \geqslant q_{-}\left(x^{2}, y^{2}, s\right)-2 \tau\left(1-r^{1 / 2}\right) \text {. }
$$

Assuming this claim for the moment, we complete the proof of the lemma. By (2.6), if $x \neq y$ and $(x, y, s) \in N_{\tau}$, we get that

$$
\begin{aligned}
\left|K_{\psi}(x, y, s)\right| & \leqslant C\|\psi\|_{\infty} \max _{0 \leqslant r<1}\left|\tilde{m}_{\alpha, r}(x, y, s)\right| \\
& \leqslant C\|\psi\|_{\infty} \max _{0 \leqslant r<1}(1-r)^{-|\alpha|-d} \exp \left(-\frac{q_{-}\left(x^{2}, y^{2}, s\right)}{1-r}\right) \Pi_{\alpha}(s) \\
& \leqslant C\|\psi\|_{\infty} q_{-}\left(x^{2}, y^{2}, s\right)^{-|\alpha|-d} \Pi_{\alpha}(s) .
\end{aligned}
$$


Moreover, if $x \neq y$, a straightforward calculation shows that, for each $i=1, \ldots, d$,

$$
\begin{aligned}
\partial_{x_{i}} \tilde{m}_{\alpha, r}(x, y, s) & =C(1-r)^{-|\alpha|-d-1}\left|x_{i} r^{1 / 2}-y_{i} s_{i}\right| r^{1 / 2} \exp \left(-\frac{q_{-}\left(r x^{2}, y^{2}, s\right)}{1-r}\right) \Pi_{\alpha}(s) \\
& \leqslant C(1-r)^{-|\alpha|-d-1 / 2} \frac{\left(q_{-}\left(r x^{2}, y^{2}, s\right)\right)^{1 / 2}}{(1-r)^{1 / 2}} \exp \left(-\frac{q_{-}\left(r x^{2}, y^{2}, s\right)}{1-r}\right) \Pi_{\alpha}(s) \\
& \leqslant C(1-r)^{-|\alpha|-d-1 / 2} \exp \left(-c_{0} \frac{q_{-}\left(r x^{2}, y^{2}, s\right)}{1-r}\right) \Pi_{\alpha}(s)
\end{aligned}
$$

and similarly

$$
\partial_{y_{i}} \tilde{m}_{\alpha, r}(x, y, s) \leqslant C(1-r)^{-|\alpha|-d-1 / 2} \exp \left(-c_{0} \frac{q_{-}\left(r x^{2}, y^{2}, s\right)}{1-r}\right) \Pi_{\alpha}(s)
$$

for some positive constant $c_{0}$. Hence, if $(x, y, s) \in N_{\tau}$ and $x \neq y$,

$$
\begin{aligned}
\left|\nabla_{(x, y)} K_{\psi}(x, y, s)\right| & \leqslant C\|\psi\|_{\infty} \max _{0 \leqslant r<1}\left|\nabla_{(x, y)} \tilde{m}_{\alpha, r}(x, y, s)\right| \\
& \leqslant C\|\psi\|_{\infty} \max _{0 \leqslant r<1}(1-r)^{-|\alpha|-d-1 / 2} \exp \left(-c_{0} \frac{q_{-}\left(x^{2}, y^{2}, s\right)}{1-r}\right) \Pi_{\alpha}(s) \\
& \leqslant C\|\psi\|_{\infty} q_{-}\left(x^{2}, y^{2}, s\right)^{-|\alpha|-d-1 / 2} \Pi_{\alpha}(s) .
\end{aligned}
$$

This concludes the proof of the lemma.

It remains to prove the claim. Let $v=\left(v_{1}, \ldots, v_{d}\right)$ and $w=\left(w_{1}, \ldots, w_{d}\right)$ be two vectors in $\mathbf{R}^{|n|}$, such that $v_{i}, w_{i} \in \mathbf{R}^{n_{i}}$ and $n_{i} \in \mathbf{N} \backslash\{\mathbf{0}\}$, for each $i=1, \ldots, d$ and $|n|=n_{1}+\cdots+n_{d}$. Suppose that $\left|v_{i}\right|=x_{i},\left|w_{i}\right|=y_{i}$ and the angle between $v_{i}$ and $w_{i}$ is $\arccos s_{i}$, for every $i=1, \ldots, d$. Then we have that $q_{ \pm}\left(x^{2}, y^{2}, s\right)^{1 / 2}=|v \pm w|$. So we get that

$$
\begin{aligned}
q_{-}\left(r x^{2}, y^{2}, s\right) & =\left|r^{1 / 2} v-w\right|^{2} \\
& \geqslant|v-w|^{2}-2\left(1-r^{1 / 2}\right) v \cdot(v-w) \\
& \geqslant q_{-}\left(x^{2}, y^{2}, s\right)-2\left(1-r^{1 / 2}\right)|x| q_{-}\left(x^{2}, y^{2}, s\right)^{1 / 2} \\
& \geqslant q_{-}\left(x^{2}, y^{2}, s\right)-2 \tau\left(1-r^{1 / 2}\right),
\end{aligned}
$$

as required.

Since Lemma 1 ensures the appropriate estimates of the function $K_{\psi}$, we may now prove that $K_{\psi}$ is the kernel of $M\left(\widetilde{\mathcal{L}}_{\alpha}\right)$ outside the diagonal.

Proposition 1. If $f$ is a test function,

$$
M\left(\tilde{\mathcal{L}}_{\alpha}\right) f(x)=\int_{\mathbf{R}_{+}^{d}} K_{\psi}(x, y) f(y) \mathrm{d} m_{\alpha}(y),
$$

for all $x$ outside the support of $f$. 
Proof: To compute the kernel of the operator $M\left(\tilde{\mathcal{L}}_{\alpha}\right)$, assume that $f$ and $g$ are test functions on $\mathbf{R}_{+}^{d}$, then

$$
\begin{aligned}
\left\langle\widetilde{\mathcal{L}}_{\alpha} r^{\tilde{\mathcal{L}}_{\alpha}} f, g\right\rangle_{\tilde{\mu}_{\alpha}} & =\left\langle r^{\tilde{\mathcal{L}}_{\alpha}} f, \widetilde{\mathcal{L}}_{\alpha} g\right\rangle_{\tilde{\mu}_{\alpha}} \\
& =\iint \tilde{m}_{\alpha, r}(x, y) f(y) \mathrm{d} m_{\alpha}(y) \overline{\widetilde{\mathcal{L}}_{\alpha} g(x)} \mathrm{d} \tilde{\mu}_{\alpha}(x) \\
& =\left\langle\widetilde{m}_{\alpha, r} \mathrm{~d} \widetilde{\mu}_{\alpha} \otimes \mathrm{d} m_{\alpha}, \widetilde{\left(\mathcal{L}_{\alpha}\right)_{x}}(\bar{g} \otimes f)\right\rangle .
\end{aligned}
$$

Here $\langle\cdot, \cdot\rangle$ denotes the pairing between distributions and test functions on $\mathbf{R}_{+}^{d} \times \mathbf{R}_{+}^{d}$, $\tilde{m}_{\alpha, r} \mathrm{~d} \widetilde{\mu}_{\alpha} \otimes \mathrm{d} m_{\alpha}$ is the distribution whose density with respect to the measure $\mathrm{d} \tilde{\mu}_{\alpha} \otimes \mathrm{d} m_{\alpha}$ is $\tilde{m}_{\alpha, r}(x, y)$, and $\left(\tilde{\mathcal{L}}_{\alpha}\right)_{x}$ denotes the operator $\tilde{\mathcal{L}}_{\alpha}$ acting in the variable $x$. Since the operator $\widetilde{\mathcal{L}}_{\alpha}$ is symmetric with respect to the Laguerre measure,

$$
\begin{aligned}
\left\langle\widetilde{\mathcal{L}}_{\alpha} r^{\tilde{\mathcal{L}}_{\alpha}} f, g\right\rangle_{\tilde{\mu}_{\alpha}} & =\left\langle\left(\widetilde{\left(\mathcal{L}_{\alpha}\right)_{x}} \widetilde{m}_{\alpha, r}\right) \mathrm{d} \widetilde{\mu}_{\alpha} \otimes \mathrm{d} m_{\alpha}, \bar{g} \otimes f\right\rangle \\
& =\iint r \partial_{r} \widetilde{m}_{\alpha, r}(x, y) \bar{g}(x) f(y) \mathrm{d} m_{\alpha}(y) \mathrm{d} \widetilde{\mu}_{\alpha}(x) .
\end{aligned}
$$

Thus, by (2.2),

$$
\left\langle M\left(\mathcal{L}_{\alpha}\right) f, g\right\rangle_{\tilde{\mu}_{\alpha}}=\int_{0}^{1} \psi(r) \iint \partial_{r} \widetilde{m}_{\alpha, r}(x, y) \bar{g}(x) f(y) \mathrm{d} \widetilde{\mu}_{\alpha}(x) \mathrm{d} m_{\alpha}(y) \mathrm{d} r
$$

If $f$ and $g$ have disjoint supports, by Lemma 1 the triple integral of the right hand side is absolutely convergent. Thus, by Fubini's theorem

$$
\left\langle M\left(\widetilde{\mathcal{L}}_{\alpha}\right) f, g\right\rangle_{\tilde{\mu}_{\alpha}}=\iint K_{\psi}(x, y) f(y) \mathrm{d} m_{\alpha}(y) \bar{g}(x) \mathrm{d} \tilde{\mu}_{\alpha}(x)
$$

This proves that $K_{\psi}$ is the restriction to the complement of the diagonal of the kernel of $M\left(\tilde{\mathcal{L}}_{\alpha}\right)$

\section{WEAK TYPE $(1,1)$ BOUNDEDNESS}

In this section we shall prove that $M\left(\widetilde{\mathcal{L}}_{\alpha}\right)$ is of weak type $(1,1)$ with respect to the measure $\tilde{\mu}_{\alpha}$. As we have seen, this is equivalent proving the $(1,1)$-estimate for $M\left(\mathcal{L}_{\alpha}\right)$ with respect to the Laguerre measure.

Now we shall decompose $M\left(\tilde{\mathcal{L}}_{\alpha}\right)$ into a "global" and "local" part. Let $\tau=8(|\alpha|+d)$ and $\varphi$ be a cut-off function on $\mathbf{R}_{+}^{d} \times \mathbf{R}_{+}^{d} \times[-1,1]^{d}$ such that $0 \leqslant \varphi \leqslant 1$,

$$
\varphi(x, y, s)= \begin{cases}1, & (x, y, s) \in N_{\tau} \\ 0, & (x, y, s) \notin N_{2 \tau}\end{cases}
$$

and

$$
\left|\nabla_{x} \varphi(x, y, s)\right|+\left|\nabla_{y} \varphi(x, y, s)\right|<\frac{c_{t}}{q_{-}\left(x^{2}, y^{2}, s\right)^{1 / 2}}
$$


Define

$$
\begin{aligned}
M^{g l} f(x) & =\int_{\mathbf{R}_{+}^{d}} \int_{[-1,1]^{d}} K_{\psi}(x, y, s)(1-\varphi(x, y, s)) \mathrm{d} s f(y) \mathrm{d} m_{\alpha}(y), \\
M^{l o c} f & =M\left(\tilde{\mathcal{L}}_{\alpha}\right) f-M^{g l} f,
\end{aligned}
$$

on the bounded measurable functions $f$. The boundedness of these operators implies the weak type $(1,1)$ of $M\left(\widetilde{\mathcal{L}}_{\alpha}\right)$. First we study $M^{g l}$.

Proposition 2. The operator $M^{g l}$ is of weak type $(1,1)$ with respect to the measure $\tilde{\mu}_{\alpha}$.

Proof: It is simple to see that, for each $f \in L^{1}\left(\tilde{\mu}_{\alpha}\right)$,

$$
\begin{aligned}
\left|M^{g l} f(x)\right| & \leqslant C\|\psi\|_{\infty} \int_{\mathbf{R}_{+}^{d}} \int_{[-1,1]^{d}} \max _{0 \leqslant r<1} \tilde{m}_{\alpha, r}(x, y, s)(1-\varphi(x, y, s)) \mathrm{d} s|f(y)| \mathrm{d} m_{\alpha}(y) \\
& \leqslant C\|\psi\|_{\infty} \int_{\mathbf{R}_{+}^{d}} \int_{[-1,1]^{d}} \max _{0 \leqslant r<1} \tilde{m}_{\alpha, r}(x, y, s)\left(1-\chi_{N_{+}}(x, y, s)\right) \mathrm{d} s|f(y)| \mathrm{d} m_{\alpha}(y) .
\end{aligned}
$$

The latter operator is of weak type $(1,1)$ by a modification of the proof of the corresponding result for the Ornstein-Uhlenbeck case, which can be found in [4] (for more details see $[\mathbf{1 0}])$.

In the following, we need the $L^{2}\left(\widetilde{\mu}_{\alpha}\right)$-boundedness of $M^{g l}$ to study $M^{\text {loc }}$. So we introduce the following result. Let $k$ be the function

$k(x, y, s)= \begin{cases}e^{-|y|^{2}}, & \sum_{i=1}^{d} x_{i} y_{i} s_{i} \leqslant 0 \\ \left(\frac{q_{+}\left(x^{2}, y^{2}, s\right)}{q_{-}\left(x^{2}, y^{2}, s\right)}\right)^{(|\alpha|+d) / 2} e^{-\left(|y|^{2}-|x|^{2}\right) / 2-\left(q_{-}\left(x^{2}, y^{2}, s\right)^{1 / 2} q_{+}\left(x^{2}, y^{2}, s\right)^{1 / 2}\right) / 2}, & \text { otherwise. }\end{cases}$

LEMMA 2. If $q_{-}\left(x^{2}, y^{2}, s\right)^{1 / 2}>(8(|\alpha|+d)) /(1+|x|+|y|)$, there exists a constant $C$ such that,

$$
\max _{0 \leqslant r<1} \tilde{m}_{\alpha, r}(x, y, s) \leqslant C k(x, y, s) \Pi_{\alpha}(s) .
$$

PRoOF: The proof is a modification to our case of the arguments used in the proof of [7, Proposition 2.1]. We omit the details.

This estimate of the kernel suffices to prove the $L^{2}\left(\tilde{\mu}_{\alpha}\right)$-boundedness of $M^{g l}$.

PRoposition 3. The operator $M^{g l}$ is bounded on $L^{2}\left(\tilde{\mu}_{\alpha}\right)$.

Proof: By Lemma 2, it is enough to verify that the operator $\mathcal{K}$ defined by

$$
\mathcal{K} f(x)=\int_{\mathbf{R}_{+}^{d}} \int_{[-1,1]^{d}} k(x, y, s) \Pi_{\alpha}(s) \mathrm{d} s f(y) \mathrm{d} m_{\alpha}(y)
$$


is bounded on $L^{2}\left(\widetilde{\mu}_{\alpha}\right)$. We may write

$$
\begin{aligned}
\mathcal{K} f(x)=\int_{\left\{\sum_{i=1}^{d} x_{i} y_{i} s_{i} \leqslant 0\right\}} k(x, y, s) \Pi_{\alpha}(s) \mathrm{d} s f(y) \mathrm{d} m_{\alpha}(y) & \\
& +\int_{\left\{\sum_{i=1}^{d} x_{i} y_{i} s_{i}>0\right\}} k(x, y, s) \Pi_{\alpha}(s) \mathrm{d} s f(y) \mathrm{d} m_{\alpha}(y) .
\end{aligned}
$$

The first integral is controlled by

$$
\int_{\mathbf{R}_{+}^{d}} \int_{[-1,1]^{d}} e^{-|y|^{2}} \Pi_{\alpha}(s) \mathrm{d} s|f(y)| \mathrm{d} m_{\alpha}(y)=C\|f\|_{L^{1}\left(\tilde{\mu}_{\alpha}\right)} \leqslant C\|f\|_{L^{2}\left(\tilde{\mu}_{\alpha}\right)} .
$$

Therefore it remains to estimate the second integral. Let $\eta \in(1 / 2,1)$ and $D_{\eta}$ be

$$
\left\{(x, y, s) \in \mathbf{R}_{+}^{d} \times \mathbf{R}_{+}^{d} \times[-1,1]^{d}: q_{-}\left(x^{2}, y^{2}, s\right)^{1 / 2}<\eta q_{+}\left(x^{2}, y^{2}, s\right)^{1 / 2}\right\} .
$$

If $\sum_{i=1}^{d} x_{i} y_{i} s_{i}>0$, then

$$
\begin{aligned}
k(x, y, s) e^{|y|^{2}} & =\frac{q_{+}\left(x^{2}, y^{2}, s\right)^{(|\alpha|+d-1) / 2}}{q_{-}\left(x^{2}, y^{2}, s\right)^{(|\alpha|+d+1) / 2}} q_{+}\left(x^{2}, y^{2}, s\right)^{1 / 2} q_{-}\left(x^{2}, y^{2}, s\right)^{1 / 2} \\
& \leqslant C \frac{q_{+}\left(x^{2}, y^{2}, s\right)^{\left(\left.||||\right|^{2}+|x|^{2}\right) / 2} e^{\left(q_{+}\left(x^{2}, y^{2}, s\right)^{1 / 2} q_{-}\left(x^{2}, y^{2}, s\right)^{1 / 2}\right) / 2}}{q_{-}\left(x^{2}, y^{2}, s\right)^{(|\alpha|+d+1) / 2}} e^{\left(|y|^{2}+|x|^{2}\right) / 2}
\end{aligned}
$$

where the last estimate follows from the fact that the function $g(t)=t e^{-t}$ is bounded on $[0,+\infty)$. We shall use the previous estimate in the region $N_{\tau}^{c} \cap D_{\eta}$.

When $\sum_{i=1}^{d} x_{i} y_{i} s_{i}>0$ and $(x, y, s) \in N_{t}^{c} \cap D_{\eta}^{c}$, it follows from the fact that

$$
\frac{1}{\eta^{2}} q_{-}\left(x^{2}, y^{2}, s\right) \geqslant q_{+}\left(x^{2}, y^{2}, s\right)>q_{-}\left(x^{2}, y^{2}, s\right),
$$

that there exists a constant $\rho$ such that

$$
k(x, y, s) e^{|y|^{2}} \leqslant C e^{\left(|y|^{2}+|x|^{2} / 2\right)-\rho q_{-}\left(x^{2}, y^{2}, s\right)} .
$$

The conclusion follows, by applying a simple adaptation of [9, Proposition 5.4] to the integral operator whose kernel is $k(x, y, s) e^{y^{2}} \chi_{N_{F}}(x, y, s)$ with respect to the measure $\tilde{\mu}_{\alpha}$.

Now to prove our main result, it is enough to get the weak type $(1,1)$ estimate for $M^{\text {loc }}$. Observe that, by (3.2), the operator $M^{\text {loc }}$ is a singular integral operator. However, $\left(\mathbf{R}_{+}^{d}, \tilde{\mu}_{\alpha}\right)$ is not a space of homogeneous type in the sense of [2], because the measure $\tilde{\mu}_{\alpha}$ is not a doubling measure. Therefore we cannot apply directly the Calderón-Zygmund theory to the operator $M^{\text {loc }}$, to prove that it is bounded on $L^{p}\left(\tilde{\mu}_{\alpha}\right)$. 
Nevertheless, we shall see that the problem may be reduced to studying the operator on $L^{p}\left(m_{\alpha}\right)$, where $m_{\alpha}$ is the measure defined in (2.4). Indeed, $\mathbf{R}_{+}^{d}$ with the measure $m_{\alpha}$ and the Euclidean distance is a space of homogeneous type.

We need to observe that there exists a collection of balls $\left\{B_{j}\right\}_{j \in \mathbf{N}}$, covering $\mathbf{R}_{+}^{d}$, such that the collection $\left\{\delta B_{j}\right\}_{j \in \mathbf{N}}$ has bounded overlap for each $\delta>1$ and on $B_{j}$ the measure $\tilde{\mu}_{\alpha}$ is equivalent to the measure $m_{\alpha}$ (see $\left[9\right.$, Lemma 5.9]), that is, there exist $C_{0}, C_{1}>0$, such that for every measurable subset $E$ of $B_{j}$

$$
C_{0} e^{-\left|x_{j}\right|^{2}} m_{\alpha}(E) \leqslant \tilde{\mu}_{\alpha}(E) \leqslant C_{1} e^{-\left|x_{j}\right|^{2}} m_{\alpha}(E) .
$$

REMARK 1. Let $\mathcal{K}$ be an operator mapping bounded measurable functions with compact support to locally integrable functions. For each $\delta>1$ fixed, define

$$
\mathcal{K}_{\ell} f=\sum_{j} \chi_{B_{j}} \mathcal{K}\left(f \chi_{\delta B_{j}}\right)
$$

for each bounded measurable function $f$. We have that if $\mathcal{K}$ extends to a bounded operator on $L^{p}\left(\widetilde{\mu}_{\alpha}\right)$ or on $L^{p}\left(m_{\alpha}\right)$ for some $p \in[1, \infty)$, then $\mathcal{K}_{\ell}$ extends to a bounded operator on $L^{p}\left(\tilde{\mu}_{\alpha}\right)$ and on $L^{p}\left(m_{\alpha}\right)$. The $L^{p}$-operator norm of $\mathcal{K}_{\ell}$ with respect to both measures, is bounded by a constant times the $L^{p}$-operator norm of $\mathcal{K}$. Moreover the same result holds for the weak type estimates $(p, p)$, with $p \in[1, \infty)$ (see [9, Lemma 5.11]).

THEOREM 1. If the function $M$ is of Laplace transform type, then the multiplier operator $M\left(\widetilde{\mathcal{L}}_{\alpha}\right)$ is of weak type $(1,1)$ with respect to the measure $\tilde{\mu}_{\alpha}$.

Proof: By Proposition 2, $M^{g l}$ is of weak type $(1,1)$. Thus by $(3.2)$, it is enough to analyse the operator $M^{\text {loc }}$. Let $\left\{B_{j}\right\}_{j \in \mathbb{N}}$ be the family of balls defined above. We shall fix $\delta>1$ later. We may write $M^{\text {loc }}$ as

$$
M^{\mathrm{loc}} f=M^{\mathrm{loc}}\left(f \chi_{\delta B_{j}}\right)+M^{\mathrm{loc}}\left(\left(1-\chi_{\delta B_{j}}\right) f\right) .
$$

By multiplying by $\chi_{B_{j}}$ and adding over $j$, we get

$$
\begin{aligned}
\left|M^{\mathrm{loc}} f\right| & \leqslant\left|\sum_{j} \chi_{B_{j}} M^{\mathrm{loc}}\left(f \chi_{\delta B_{j}}\right)\right|+\left|\sum_{j} \chi_{B_{j}} M^{\mathrm{loc}}\left(\left(1-\chi_{\delta B_{j}}\right) f\right)\right| \\
& :=\left|M_{\ell}^{\mathrm{loc}} f\right|+|R f| .
\end{aligned}
$$

By spectral theory and Proposition $3, M\left(\tilde{\mathcal{L}}_{\alpha}\right)$ and $M^{g l}$ are bounded on $L^{2}\left(\tilde{\mu}_{\alpha}\right)$. It follows that $M^{\text {loc }}$ is bounded on $L^{2}\left(\widetilde{\mu}_{\alpha}\right)$. So by Remark $1, M_{\ell}^{\text {loc }}$ is bounded on $L^{2}$ with respect to both measures, $m_{\alpha}$ and $\tilde{\mu}_{\alpha}$. We claim that $R$ is bounded both on $L^{p}\left(\tilde{\mu}_{\alpha}\right)$ and on $L^{p}\left(m_{\alpha}\right)$, for $1 \leqslant p \leqslant \infty$. Assuming this claim for the moment, we complete the proof. The operator $M^{\text {loc }}$ is an integral singular operator, whose kernel is $\int_{[-1,1]^{d}} K_{\psi}(x, y, s) \varphi(x, y, s) \mathrm{d} s$, outside the diagonal. By the claim, $M^{\text {loc }}$ is bounded on $L^{2}\left(m_{\alpha}\right)$. Moreover, by (3.1) and Lemma 1

$$
\left|\nabla_{(x, y)}\left[K_{\psi}(x, y, s) \varphi(x, y, s)\right]\right| \leqslant C\|\psi\|_{\infty} q_{-}\left(x^{2}, y^{2}, s\right)^{-|\alpha|-d-1 / 2} \Pi_{\alpha}(s)
$$


for each $(x, y, s) \in N_{2 \tau}$ and $x \neq y$. Hence by an extension of the classical CalderónZygmund theory for the singular operator on $\left(\mathbf{R}_{+}^{d}, m_{\alpha}\right)$ (see [9, Proposition 5.7]), $M^{\text {loc }}$ is of weak type $(1,1)$ with respect to the measure $m_{\alpha}$. By Remark $1, M_{\ell}^{\text {loc }}$ is of weak type $(1,1)$ with respect to the measure $\widetilde{\mu}_{\alpha}$ and

$$
\left\|M^{\text {loc }} f\right\|_{L^{1, \infty}\left(\tilde{\mu}_{\alpha}\right)} \leqslant\left\|M_{\ell}^{\text {loc }} f\right\|_{L^{1, \infty}\left(\tilde{\mu}_{\alpha}\right)}+\|R f\|_{L^{1}\left(\tilde{\mu}_{\alpha}\right)},
$$

as desired. In order to conclude it is necessary to prove the claim. Note that the kernel with respect to the measure $m_{\alpha}$ of the operator $R$ is

$$
H(x, y)=\sum_{j} \chi_{B_{j}}(x) \int_{[-1,1]^{d}} K_{\psi}(x, y, s) \varphi(x, y, s) \mathrm{d} s\left(1-\chi_{\delta B_{j}}(y)\right) .
$$

We may choose $\delta>1$ such that the function

$$
(x, y, s) \mapsto \sum_{j} \chi_{B_{j}}(x) K_{\psi}(x, y, s) \varphi(x, y, s)\left(1-\chi_{\delta B_{j}}(y)\right),
$$

is supported in $N_{2 \tau} \backslash N_{\tau}$. Indeed, it is quite straightforward to see that, for $t$ fixed, there exists $\delta>1$ such that if $x \in B_{j}$ and $y \notin \delta B_{j}$, then $(x, y, s) \notin N_{t}$ for each $s \in[-1,1]^{d}$ (see $\left[10\right.$, Remark 2.29]). Thus, for $(x, y, s) \in N_{2 \tau} \backslash N_{\tau}$, by Lemma 1

$$
\left|K_{\psi}(x, y, s)\right| \leqslant C q_{-}\left(x^{2}, y^{2}, s\right)^{-|\alpha|-d} \Pi_{\alpha}(s)<C(1+|x|+|y|)^{2(|\alpha|+d)} \Pi_{\alpha}(s) .
$$

Moreover

$$
|x-y| \leqslant q_{-}\left(x^{2}, y^{2}, s\right)^{1 / 2}<\frac{2 \tau}{1+|x|+|y|} .
$$

Hence $|H(x, y)| \leqslant C(1+|y|)^{2|\alpha|+2 d} \int_{[-1,1]^{d}} \chi_{N_{i}^{c}}(x, y, s) \Pi_{\alpha}(s)$ d $s$. The boundedness of $R$ on $L^{q}$, with $1 \leqslant q \leqslant \infty$, for both measures, follows from these estimates, as in the proof of $[9$, Proposition 5.1 (19)].

REMARK 2. Now if $M$ is of Laplace transform type, the weak type $(1,1)$ of $M\left(\mathcal{L}_{\alpha}\right)$ with respect to the Laguerre measure follows easily by Theorem 1.

\section{REFERENCES}

[1] U. Dinger, 'Weak type $(1,1)$ estimates of the maximal function for the Laguerre semigroup in finite dimensions', Rev. Mat. Iberoamericana 8 (1992), 93-118.

[2] R.R. Coifman and G. Weiss, Analyse harmonique non-commutative sur certains espaces homogènes, Lecture Notes in Mathematics 242 (Springer-Verlag, Berlin, Heidelberg, New York, 1971).

[3] C. Gutiérrez, A. Incognito and J. Torrea, 'Riesz Transforms, $g$-functions and multipliers for Laguerre semigroup', Houston J. Math. 27 (2001), 579-592.

[4] J. Garcia-Cuerva, G. Mauceri, S. Meda, P. Sjögren and J. Torrea, 'Maximal operators for the holomorphic Ornstein-Uhlenbeck semigroup', J. London Math. Soc. (2) 67 (2003), 219-234. 
[5] J. Garcia-Cuerva, G. Mauceri, P. Sjögren and J. Torrea, 'Spectral multipliers for the Ornstein-Uhlenbeck semigroup', J. Analyse Math. 78 (1999), 281-305.

[6] S. Meda, 'A general multiplier theorem', Proc. Amer. Math. Soc. 110 (1990), 639-647.

[7] T. Menárguez, S. Pérez and F. Soria, 'The Mehler maximal function: a geometric proof of the weak type 1', J. London Math. Soc. (2) 61 (2000), 846-856.

[8] B. Muckenhoupt, 'Poisson integral for Hermite and Laguerre expansions', Trans. Amer. Math. Soc. 139 (1969), 231-242.

[9] E. Sasso, 'Functional calculus for the Laguerre operator', (preprint 2003).

[10] E. Sasso, Functional calculus and maximal operator associated to the Laguerre semigroup, Ph.D.-thesis (Universitá di Genova, Italy, 2003).

[11] E.M. Stein, Topics in harmonic analysis related to the Littlewood-Paley theory, Annals of Math. Study 63 (Princeton Univ. Press, Princeton, N.J., 1970).

[12] G. Szegö, Orthogonal polynomials (Revised Edition), American Mathematical Society Colloquium Publications 23 (American Mathematical Society, Providence, R.I., 1959).

Dipartimento di Matematica

Universita di Genova

via Dodecanesco 35

16146 Geonva

Italy 